\title{
2D Shape Classification Using Multifractional Brownian Motion
}

\author{
Manuele Bicego and Alessandro Trudda \\ DEIR - University of Sassari \\ via Torre Tonda, 34 - 07100 Sassari (Italy) \\ \{bicego, atrudda\}@uniss.it
}

\begin{abstract}
In this paper a novel approach to contour-based 2D shape recognition is proposed. The main idea is to characterize the contour of an object using the multifractional Brownian motion $(\mathrm{mBm})$, a mathematical method able to capture the local self similarity and long-range dependence of a signal. The $\mathrm{mBm}$ estimation results in a sequence of Hurst coefficients, which we used to derive a fixed size feature vector. Preliminary experimental evaluations using simple classifiers with these feature vectors produce encouraging results, also in comparison with the state of the art.
\end{abstract}

\section{Introduction}

Object recognition represents an important research area in computer vision and pattern recognition. The classification of three-dimensional (3D) objects has been faced by different approaches [12, many of which are based on the analysis of two-dimensional (2D) aspects of objects (2D shapes). In this context, the analysis could be performed by analyzing the boundary (external approaches) or the whole shape (internal approach). In the former context, many contour descriptors have been proposed, like, for example, Fourier descriptors and chain code, whereas, in the latter context features like the medial axis, or moments, have been employed [2. In particular, object contours proved to be very effective in many applications, with many different approaches being presented over the past years, each with different characteristics, like robustness to noise and occlusions, invariance to translation, rotation and scale, computational requirements, and accuracy [23].

In this paper a novel approach to contour-based 2D shape recognition is proposed. The main idea is to characterize the contour of each object using the multifractional Brownian motion $(\mathrm{mBm})$ [456], a mathematical method employed in the finance community to characterize and model the financial series. The simplest way of introducing the multifractional Brownian motion is to consider it as an extension of the fractional Brownian motion (fBm) [7, a process characterized by a slowly decaying autocorrelation function depending on the parameter $H \in(0,1]$ (named Hurst exponent). The fBm may model the self similarity and long-range dependence of a process. The Hurst exponent $H$ has 
a clear interpretation: if $H=1 / 2$, then the $\mathrm{fBm}$ represents the standard Brownian motion (namely the process has no memory); for $H>1 / 2$, the process is characterized by a persistence (positive or negative) - namely the process has a clear trend; finally, for $H<1 / 2$ the process is characterized by an antipersistence. Even if this method has been largely applied in the finance community (mainly to understand some characteristics of the financial series), some works applying the $\mathrm{fBm}$ function have also been presented in the pattern recognition context, like in medical imaging [8] or in speech recognition [9]. It is important to note that $\mathrm{fBm}$ is related to the fractal theory [10 - a theory largely employed in image analysis [11] or even object classification [1213] - since it is modeling self-similarity. Actually it can be shown that, for fractal or self-similar processes, the Horst exponent $H$ can be related to a fractal dimension $D_{h}$ [10] through the equation $D_{h}=2-H$, where $D_{h}$ is the fractal Hausdorff dimension [14].

Nevertheless, from a shape recognition point of view, we consider it too strong to assume contours showing a complete self similarity, and modeling them with a fBm process (or fractals) could be too approximate. Still, the description power of such models may be successfully applied if we model local self-similarity, that is by considering objects as self similar in limited region. This behavior could be modeled by the so called multifractional Brownian motion $(\mathrm{mBm})$, an extension of fBm so that the Hurst exponent can vary over time. As a result of this process, a function $H(t)$ is obtained, describing the self similarity of the process in a window centered in $t$. The $\mathrm{mBm}$ process is no longer stationary nor self-similar; despite this, the process is extremely versatile since the time dependency of $H$ is useful to model phenomena whose punctual regularity is time changing, this characteristic being possibly really useful in characterizing an object's shape.

In this paper we explore the use of the $\mathrm{mBm}$ function for characterizing the shape contours with the aim of $2 \mathrm{D}$ shape classification. In the proposed approach, the contour is modeled with the curvature (similarly to [1516]), which was fitted with a mBm function. The sequence of Horst coefficients computed was subsequently employed to derive a fixed length vector, which characterizes each shape. Some experimental evaluations were performed using simple Nearest Neighbor classifier (with the Euclidean and the Minkowsky distance) on the Chicken database, showing promising results also in comparison with the state of the art.

\section{The Multifractional Brownian Motion (mBm) Approach}

This section will present the theory of the $\mathrm{mBm}$ approach, as well as the estimation methodology we used here.

\subsection{The mBm Model}

As reported in the introduction, a convenient way to introduce the $\mathrm{mBm}$ is to consider it as an extension of the fractional Brownian motion (fBm). Defined in a 
celebrated paper by Mandelbrot and Van Ness [7], the fBm is characterized by a slowly decaying autocorrelation function depending on the parameter $H \in(0,1]$, named Hurst exponent. Following the definition that can be found in [17, the process has the following moving average representation

$$
B_{H}(t)=C\{\pi K(2 H)\}^{1 / 2} \int_{\mathbb{R}} f_{t}(s) d B(s)
$$

with

$$
f_{t}(s)=\frac{1}{\Gamma\left(H+\frac{1}{2}\right)}\left\{|t-s|^{H-\frac{1}{2}} 1_{]-\infty, t]}(s)-|s|^{H-\frac{1}{2}} 1_{]-\infty, 0]}(s)\right\}
$$

where $C$ is a positive constant, $K$ is the function defined on $] 0,2[$ as $K(\alpha)=$ $\Gamma(\alpha+1) \frac{\sin \frac{\alpha \pi}{2}}{\pi}$, and $B(\cdot)$ stands for the ordinary Brownian motion process introduced by Robert Brown in 1872 to describe the random movements of particles suspended in a liquid or gas 18 . The process is self-similar 1 of parameter $H$ and has stationary increments. Its covariance function reads as

$$
E\left(B_{H}(t) B_{H}(s)\right)=\frac{c^{2}}{2}\left(|t|^{2 H}+|s|^{2 H}-|t-s|^{2 H}\right)
$$

The fBm can be generalized by allowing $H$ to vary over time. This extension - known as multifractional Brownian motion $(\mathrm{mBm})$ [45] - has the following representation

$$
M_{H(t)}(t)=C\{\pi K(2 H(t))\}^{1 / 2} \int_{\mathbb{R}} f_{t}(s) d B(s)
$$

with

$$
f_{t}(s)=\frac{1}{\Gamma\left(H(t)+\frac{1}{2}\right)}\left\{|t-s|^{H(t)-\frac{1}{2}} 1_{]-\infty, t]}(s)-|s|^{H(t)-\frac{1}{2}} 1_{]-\infty, 0]}(s)\right\}
$$

where $H:[0, \infty) \rightarrow(0,1]$ is required to be a Hölder function of order $0<\eta \leq 1$ to ensure the continuity of the motion.

Notice that since $H(t)$ is the punctual Hölder exponent of the $\mathrm{mBm}$ at point $t$, the process is locally asymptotically self-similar with index $H(t)$ (see, e.g. [20] in the sense that, denoted by $Z(t, a u):=M_{H(t+a u)}(t+a u)-M_{H(t)}(t)$ the increment process of the $\mathrm{mBm}$ at time $t$ and lag $a u$, it holds

$$
\lim _{a \rightarrow 0^{+}} a^{-H(t)} Z(t, a u) \stackrel{d}{=} B_{H(t)}(u), u \in \mathbb{R} .
$$

\footnotetext{
${ }^{1}$ We recall that the process $\{X(t), t \in T\}$ is said self-similar with parameter $H$ if for any $\alpha>0\{X(\alpha t)\} \stackrel{d}{=}\left\{\alpha^{H} X(t)\right\}$, where the equality holds for the finite-dimensional distributions of the process (see e.g. [19]).
} 
The above distributional equality indicates that at any point $t$ there exists an $\mathrm{fBm}$ with parameter $H(t)$ tangent to the $\mathrm{mBm}$. Moreover, since $B_{H(t)}(u) \sim$ $\mathcal{N}\left(0, C^{2} u^{2 H(t)}\right)$, the infinitesimal increment of the $\mathrm{mBm}$ at time $t$, normalized by $a^{H(t)}$, normally distributes with mean 0 and variance $C^{2} u^{2 H(t)}\left(u \in \mathbb{R}, a \rightarrow 0^{+}\right)$. The increments of the $\mathrm{mBm}$ are no longer stationary nor self-similar; despite this, the process is extremely versatile since the time dependency of $H$ is useful to model phenomena whose punctual regularity is time changing.

\subsection{Point-Wise Estimation of the Mbm}

One of the main problems when using the $\mathrm{mBm}$ process is to estimate the function $H(t)$ from actual data. To face this problem one could think to adapt the traditional estimators of $H$ available in literature in order to shadow the dynamics of $H(t)$. The weakness of this approach resides in the fact that very large samples are needed to get reliable estimates and over a long period of time $H$ is likely to change even further. So, more efficient estimators are needed in the case of the $\mathrm{mBm}$. An answer to this problem is provided by Bianchi in [21], who develops the work of 22 and defines a family of "moving-window" estimators of $H(t)$ based on the $k$-th absolute moment of a Gaussian random variable of mean zero and given variance $V_{H}$ (the variance of the unit lag increment of a $\mathrm{mBm})$. Given a series of length $N$ and a window of length $\delta$, the estimator has the form

$$
\begin{aligned}
H_{\delta, N}^{k}(t) & =\frac{\log \left(2^{k / 2} \Gamma\left(\frac{k+1}{2}\right) V_{H}^{k / 2}\right)-\log \left(\frac{\sqrt{\pi}}{\delta} \sum_{j=t-\delta}^{t-1}\left|X_{j+1, N}-X_{j, N}\right|^{k}\right)}{k \log (N-1)} \\
\text { for } j & =t-\delta, \ldots, t-1 ; \quad t=\delta+1, \ldots, N ; \quad k \geq 1 .
\end{aligned}
$$

When $H \in\left(0, \frac{3}{4}\right)$, the estimators' rate of converge is $O\left(\delta^{-\frac{1}{2}}(\log N)^{-1}\right)$, what allows to get reliable estimates even for very short $\delta^{\prime} s$.

The family of estimators (5) was proved to be correct and normally distributed as

$$
H_{\delta, N}^{k}(t) \sim \mathcal{N}\left(H(t), \frac{\pi}{\delta k^{2} \ln ^{2}(N-1) 2^{k}\left(\Gamma\left(\frac{k+1}{2}\right)\right)^{2}} \sigma^{2}\right) .
$$

$\sigma^{2}$ being the variance of a Gaussian random variable defined as a proper rescaled sum. Toilsome computations show that when $H=\frac{1}{2}$ the variance of the estimator reduces to

$$
\operatorname{Var}\left(H_{\delta, N}^{k}(t)\right)=\frac{\sqrt{\pi}}{\delta k^{2} \ln ^{2}(N-1)\left[\Gamma\left(\frac{k+1}{2}\right)\right]^{2}} \cdot\left(\Gamma\left(\frac{2 k+1}{2}\right)-\frac{1}{\sqrt{\pi}}\left[\Gamma\left(\frac{k+1}{2}\right)\right]^{2}\right)
$$


and the optimal value of $k$ is deduced by minimizing the last relation. So one finds that the minimum of (8) takes place when $k=2$, value which will be used in the experiments discussed below.

\section{The Proposed Approach}

In this section the proposed approach is summarized: first the contours are encoded in one-dimensional signals, then the $\mathrm{mBm}$ is estimated and a feature vector is extracted from $H(t)$.

\subsection{Coding of Contours}

Each object is represented by the sequence of the curvature coefficients, computed as described in 1516. Curvature has been chosen since it presents many different appealing characteristics, like invariance to translation and somehow to rotation (it results just in a shift of the signal). In order to compute curvature sequence, the contours are firstly extracted by using the Canny edge detector; the boundary is then approximated by segments of approximately fixed length $d_{L}$. Finally, the curvature value at point $x$ is computed as the angle between the two consecutive segments intersecting at $x$. The initial point is the leftmost point lying on the horizontal line passing through the object centroid, following the boundary in a counterclockwise manner.

\subsection{Feature Extraction}

It should be noted that, in the finance community, the main goal is to approximate the series with the $\mathrm{mBm}$ process, in order to gain knowledge from the sequence of Hurst coefficients and understand the persistence or anti-persistence behavior of the financial series. Here the goal is different: we want to approximate the contour signal with the $\mathrm{mBm}$ function, subsequently extracting a feature vector from the sequence $H(t)$ used to characterize the shape.

In particular each object's curvature sequence is modeled with a $\mathrm{mBm}$ process and a function $H(t)$ is extracted. It is worthwhile to observe that the signal $H(t)$ could be used as it is as a signature of the shape, with many attractive characteristics (one for all: rotation of the object simply results in a shift of the curvature and consequently only a shift of the $H(t))$. Nevertheless, in this preliminary study, we opted for a more simple and compact solution: the feature vector was obtained by sub-sampling $H(t)$ with a regular step (this has a clear impact on the rotation-invariance); in order to obtain exactly the same number of points for each shape, the sub-sampling step varies with the length of the sequence. In order to increase the robustness, the sub-sampling was carried out by averaging the signal in a small interval around the sampling points.

To summarize, there are two crucial parameters in the proposed system: the window size $\delta$ (lag), defining the "scale" of the analysis, and the number of sampled points, defining the trade-off between the capacity of the descriptor 

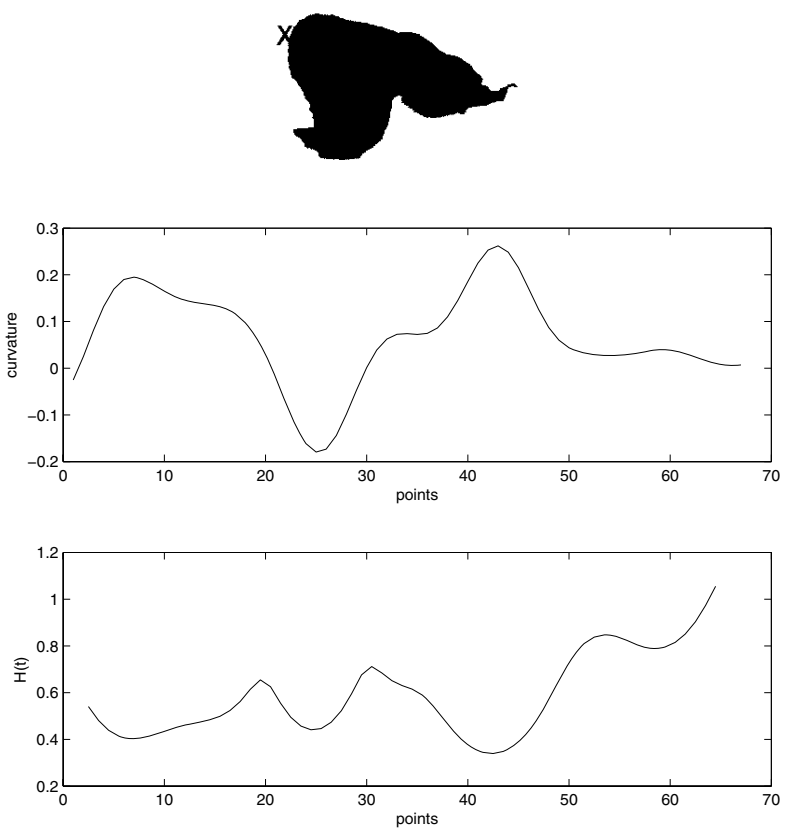

Fig. 1. (Top): one sample object; (center): curvature extracted from the object contour - starting from the "X" and following the boundary in a counterclockwise manner; (bottom) the estimated $H(t)$, computed with a lag $\delta=5$. The value of $H(t)$ at a certain point $x$ derives from the application of the estimator of $H$ to the subwindow of the signal centered in $x$.

and the curse of dimensionality. In the experimental part, we made a thorough analysis of the accuracy of the descriptors when varying these parameters.

An example of the application of the above described approach is displayed in Fig. 1] the curvature signal (middle) of the contour of the object (top) is approximate with a $\mathrm{mBm}$, which $H(t)$ is displayed in the bottom part. It is clear how this $H(t)$ encodes the regularities/indentations of the signal: where the signal has a change in the regularity (at points 25 and 45 - corresponding to the abrupt change in the contour) $H(t)$ suddenly decreases - modeling a somehow turbulent behavior. On the other side, when the behavior is regular (zone 50-70), $H(t)$ suddenly approaches 1 .

\section{Experimental Results}

We chose to study the Chicken Pieces Database2, denoted also as Chicken data [23. This set consists of 446 binary images of chicken pieces, whose examples are shown in Fig. 2, Each piece belongs to one of the five classes, representing

$\overline{2}$ See http://algoval.essex.ac.uk:8080/data/sequence/chicken/. 


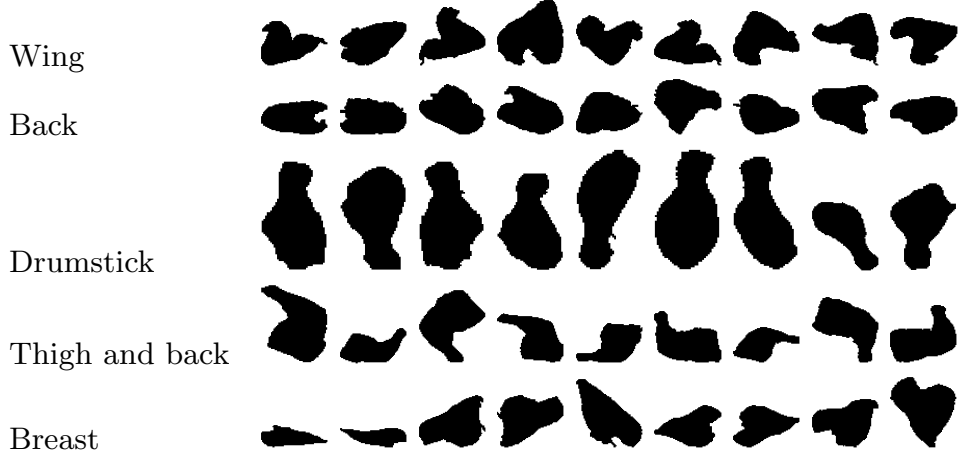

Fig. 2. Examples from the Chicken data

specific parts of a chicken: wing (117 samples), back (76), drumstick (96), thigh and back (61), and breast (96). The shapes are usually first described by contours, which are further encoded by suitable sequences. This poses a difficult classification task. The results published in 24 report a baseline leave-one-out (LOO) accuracy of $\approx 67 \%$ by using the $1-\mathrm{NN}$ on the Levenshtein (non-cyclic) edit distance computed on the contour chain code.

The sequences of the dataset were encoded using the proposed approach in order to obtain a feature space. In the feature space any classifier may be used. In order to have a clear idea of the discriminative capability of the features we used a very simple one, namely the Nearest Neighbor. We computed the leave-oneout (LOO) accuracy using the proposed approach. Two different vector-based distances were employed in the feature space: the classic Euclidean distance and the Minkowsky distance. We let the parameter $\delta$ vary from 2 to 25 , whereas the dimensionality of the feature vector was analyzed in the range [2-10]. The results are graphically shown in Fig. 3, while increasing window size $\delta$. For each $\delta$, we chose the dimensionality of the feature vector rising to the best accuracy.

From the figure it could be noted that results are quite satisfactory, even if the task is extremely difficult. Results with Euclidean Distance seem to be slightly better than those with Minkowski distance. It may also be noted that the results seem to improve when increasing the analysis window size (actually the best result was obtained with $\delta=20$ with a $76.5 \%$ of accuracy). This is true up to a certain point: when increasing too much the $\delta$, the system performances start to decrease, mainly because the $\mathrm{mBm}$ will consider larger parts of the shape as self similar (so, to some extent, leading to the fBm).

\subsection{Comparative Analysis}

This database has been widely used in the literature, with different representation and different classifiers. Since our goal is to compare the discriminative power of the proposed features, here we have reported results of other methods which only employ simple classifiers, like the Nearest Neighbor (or the K-Nearest 


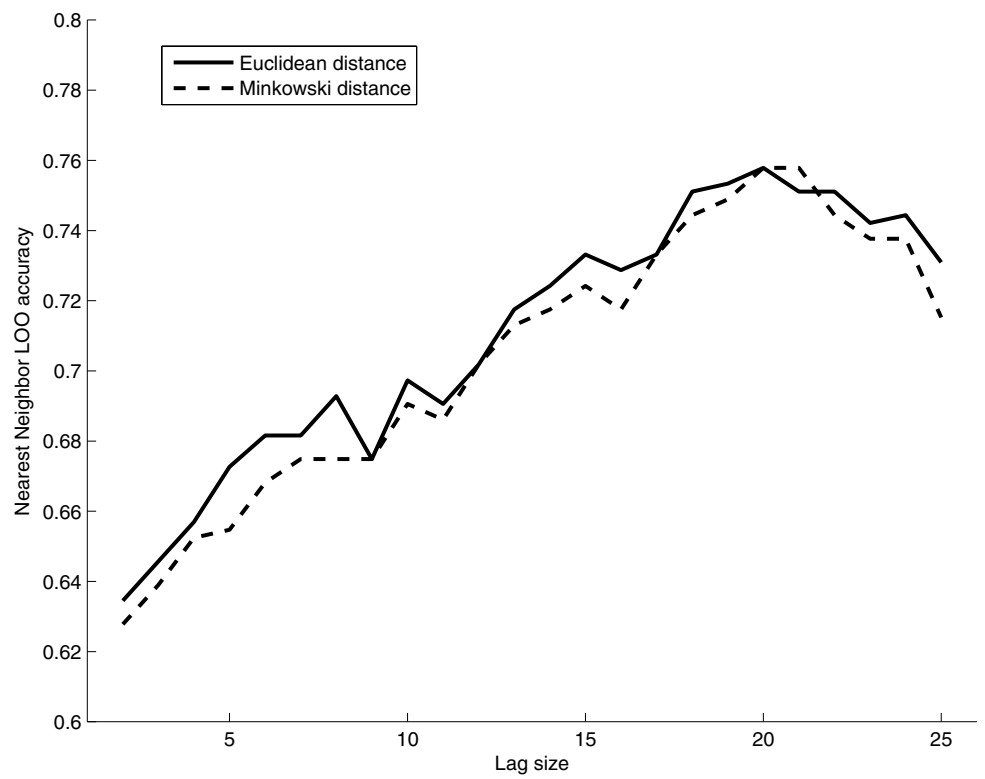

Fig. 3. LOO accuracy using the NN classifier in the proposed feature space

Neighbor). Some of them are taken from literature, some of them have been computed by the authors.

1. Fractional Brownian motion (fBm): naturally the first comparison is obtained by applying the simpler fBm to the sequences extracted from the shapes. As explained in the previous sections, this representation in too simplistic and not suitable for describing a shape. This is confirmed by experimental evidence, since the LOO accuracy using NN classifier (and Euclidean Distance) was really poor, about $38.79 \%$.

2. In 24, a structural approach has been applied to this problem, with a cyclic string matching procedure. Many different techniques have been proposed: as said before, the Levenshtein (non-cyclic) edit distance computed on the contour chain code returns a LOO error (with Nearest Neighbor) of about $67 \%$, whereas some extensions and improvements arrive up to $78 \% 3$

3. Another structural approach has been proposed by Neuhaus and Bunke in [16] where a kernel based method, based on a dissimilarity representation, was proposed. As a reference system, given the sequences encoded with curvature coefficients - similarly to what done here - they compute the accuracy of a K-Nearest Neighbor where the distances were determined using an

${ }^{3}$ Approximate values are shown since in the paper no numerical values were given, just a graphical table - Tab. 5(b). 
efficient cyclic string edit distance algorithm [25]. The accuracy, computed by splitting the set in Training/Testing/Validation was 74.3\%4.

4. Hidden Markov Models: recognition of $2 \mathrm{D}$ shapes is a quite unconventional application of HMMs, even though promising results have been reported 26 27 15. The conventional approach is train one HMM for each class, subsequently classifying with the standard Bayes rule. Nevertheless, since the tackled dataset presents a notably high intra-class variability - and a single HMM per class would be too general and so not able to solve the task - we trained one model for each training sequence, assign an unknown sequence to the class of the model showing the highest likelihood (similarly to what was done in [28]): clearly this may be seen as a 1-nearest-neighbor (1-NN) classifier, with the proximity measure defined by the likelihood function. After a preliminary evaluation, HMMs are trained with $K=3$ states for all classes. The LOO error computed was of $73.77 \%$.

From the previous results we may observe the suitability of the proposed representation technique, also in comparison with the state of the art.

\section{Conclusions}

In this paper a novel 2D shape descriptor has been proposed, which characterizes the contour of each object using the multifractional Brownian motion $(\mathrm{mBm})$. This function represents a mathematical method able to capture the local self similarity and long-range dependence of the silhouette of an object. The $\mathrm{mBm}$ estimation results in a sequence of Hurst coefficients, which we used to derive a fixed size feature vector. Preliminary experimental evaluations using simple classifiers on these feature vectors produced encouraging results.

Current research directions are mainly directed toward two goals: first, the automatic selection of the dimension of the window size $\delta$ - this possibly being inferrable from a local R/S analysis; second, a more effective classification scheme able to exploit the description power of the proposed features. Clearly, a more thorough experimental evaluation is also needed, involving different datasets and different classifiers.

\section{References}

1. Campbell, R., Flynn, P.: A survey of free-form object representation and recognition techniques. Computer Vision and Image Understanding 81, 166-210 (2001)

2. Loncaric, S.: A survey of shape analysis techniques. Pattern Recognition 31(8), 983-1001 (1998)

3. Suetens, P., Fua, P., Hanson, A.: Computational strategies for object recognition. ACM Compuiting Surveys 24(1), 5-61 (1992)

4. Peltier, R., Véhel, J.L.: Multifractional brownian motion: definition and preliminary results. Technical Report Rapport de recherche no. 2645, INRIA (1995)

\footnotetext{
${ }^{4}$ With the kernel based method they were able to reach $81.1 \%$ of accuracy.
} 
5. Véhel, J.L.: Fractal approaches in signal processing. Fractals 3, 755-775 (1995)

6. Ayache, A., Véhel, J.L.: The generalized multifractional brownian motion. Statistical Inference for Stochastic Processes 3, 7-18 (2000)

7. Mandelbrot, B., Ness, J.V.: Fractional brownian motions, fractional noises and applications. SIAM Review 10, 422-437 (1968)

8. Lundahl, T., Ohley, W., Kay, S., Siffert, R.: Fractional brownian motion: A maximum likelihood estimator and its application to image texture. IEEE Trans. on Medical Imaging 5, 152-161 (1986)

9. SantAna, R., Coelho, R., Alcaim, A.: Text-independent speaker recognition based on the hurst parameter and the multidimensional fractional brownian motion model. IEEE Trans. on Audio, Speech and Language Processing 14(3) (2006)

10. Mandelboot, B.B., et al.: The Science of Fractal Images. Springer, New York (1988)

11. Chen, S., Keller, J., Crownover, R.: On the calculation of fractal features from images. IEEE Trans. om Pattern Analysis and Machine Intelligence 15 (1993)

12. Taylor, R., Lewis, P.: 2d shape signature based on fractal measurements. IEE Proc. of Vision, Image and Signal Processing 141(6), 422-430 (1994)

13. Uemura, S., Haseyama, M., Kitajima, H.: Efficient contour shape description by using fractal interpolation functions. In: Proc. Int. Conf. Image Processing, vol. 1, pp. I-485-I-488 (2002)

14. Falconer, K.: Fractal Geometry: Mathematical Foundations and Applications. Wiley, Chichester (2003)

15. Bicego, M., Murino, V.: Investigating Hidden Markov Models' capabilities in 2D shape classification. IEEE Trans. on Pattern Analysis and Machine Intelligence PAMI 26(2), 281-286 (2004)

16. Neuhaus, M., Bunke, H.: Edit distance-based kernel functions for structural pattern classification. Pattern Recognition 39, 1852-1863 (2006)

17. Coeurjolly, J.: Identification of the multifractional brownian motion. Bernoulli 11(6), 987-1008 (2005)

18. Brown, R.: A brief account of microscopical observations made in the months of June, July and August, 1827, on the particles contained in the pollen of plants; and on the general existence of active molecules in organic and inorganic bodies. Phil. Mag. 4(1828), 161-173

19. Samorodnitsky, G., Taqqu, M.: Stable non-Gaussian random processes. Chapman \& Hall, Boca Raton (1994)

20. Benassi, A., Jaffard, S., Roux, D.: Elliptic gaussian random processes. Matematica Iberoamericana 13(1), 19-89 (1997)

21. Bianchi, S.: Pathwise identification of the memory function of the multifractional brownian motion with application to finance. Int. J. of Theoretical and Applied Finance 8(2), 255-281 (2005)

22. Peltier, R., Véhel, J.L.: A new method for estimating the parameter of fractional brownian motion. Technical Report Rapport de recherche no. 2396, INRIA (1994)

23. Andreu, G., Crespo, A., Valiente, J.: Selecting the toroidal self-organizing feature maps (tsofm) best organized to object recognitio. In: Proc. of IEEE ICNN 1997, vol. 2, pp. 1341-1346 (1997)

24. Mollineda, R., Vidal, E., Casacuberta, F.: Cyclic sequence alignments: Approximate versus optimal techniques. Int. Journal of Pattern Recognition and Artificial Intelligence 16(3), 291-299 (2002)

25. Peris, G., Marzal, A.: Fast cyclic edit distance computation with weighted edit costs in classification. In: Proc. of Int. Conf. on Pattern Recognition, vol. 4, pp. 184-187 (2002) 
26. He, Y., Kundu, A.: 2-D shape classification using Hidden Markov Model. IEEE Trans. Pattern Analysis Machine Intelligence 13(11), 1172-1184 (1991)

27. Arica, N., Yarman-Vural, F.: A shape descriptor based on circular Hidden Markov Model. In: IEEE Proc. Int. Conf. Pattern Recognition, vol. 1, pp. 924-927 (2000)

28. Bicego, M., Murino, V., Figueiredo, M.: Similarity-based classification of sequences using hidden markov models. Pattern Recognition 37(12), 2281-2291 (2004) 